\title{
Integrated assessment of water resources: Australian experiences
}

\author{
B. F. W. Croke · J. L. Ticehurst - R. A. Letcher • \\ J. P. Norton - L.T. H. Newham - A. J. Jakeman
}

Received: 20 April 2006

(C) Springer Science + Business Media B.V. 2006

\begin{abstract}
It is widely accepted that water resource management demands an integrated assessment of resource use options, including local and regional impacts on the environment and stakeholders. Multiple issues, stakeholders and scales of system behaviour must be considered, as well as the key disciplines within and between the human and natural sciences. Modelling is a critical tool in integrated assessment. It enables effects of policy interventions, climate forcing and demographics to be predicted (although with some uncertainty), and provides a means of expanding understanding of river basin behaviour. It also acts as a vehicle for social learning among various interest groups. This paper discusses the various frameworks and methods being used for integrated modelling, and their suitability and unfulfilled potential for these purposes. The frameworks include coupled component models, systems dynamics models, metamodels, risk-assessment approaches, Bayesian decision networks, agent-based methods, expert systems and other heuristic knowledge-based techniques. Specific software platforms are not considered but the lessons from software development and implementation are clearly spelt out. The paper presents three Australian case studies in integrated assessment. They vary in their range of catchment/watershed sizes, hydroclimatology, issues of concern and stakeholders engaged. Two of them utilise a coupled component modelling framework and the third a Bayesian decision network approach. The paper illustrates the value, problems and lessons of integrated assessment and modelling. In particular it proposes some
\end{abstract}

\footnotetext{
J. L. Ticehurst · L. T. H. Newham

Integrated Catchment Assessment and Management (iCAM) Centre, The Australian National University, Canberra

B. F. W. Croke $(\bowtie) \cdot$ R. A. Letcher · J. P. Norton

Integrated Catchment Assessment and Management (iCAM) Centre, and Department of Mathematics, The Australian National University, Canberra e-mail: barry.croke@anu.edu.au
}

\section{A. J. Jakeman}

Integrated Catchment Assessment and Management (iCAM) Centre, and Centre for Resource and Environmental Studies, The Australian National University, Canberra 
ways to address the challenges of assessing options to obtain more sustainable basin-wide outcomes.

Keywords Integration frameworks · System dynamics $\cdot$ Metamodels $\cdot$ Risk assessment · Bayesian decision networks · Agent-based $\cdot$ Expert systems · Knowledge-based · Integrated modelling $\cdot$ Stakeholders $\cdot$ River basin management

\section{Introduction}

Integrated management of a catchment, watershed or river basin requires integration (i.e. joint consideration) of: (i) different objectives and their related outcomes, e.g. economic efficiency, social equity and ecological integrity; (ii) all water resources (surface water, groundwater, estuaries); (iii) multiple water- and land-related issues; (iv) different types of water use (agricultural, ecological, domestic, industrial, recreational); (v) all water users, everyone affected by water use and interest groups generally. For such complex integration, modelling must play a key facilitating role, whatever its form: qualitative or quantitative, traditional or novel, or typically a mix of these forms. It must meet the challenge of representing, but not over-representing, the complexity and spatial and temporal variation within catchments and river basins. The modelling must cover a range of sciences including. hydrology, ecology, agriculture, forestry, economics, demography, psychology, sociology and politics, and a range of categories of people affected. The evolving discipline of Integrated Assessment (IA) aims to deal with such situations. According to The Integrated Assessment Society (www.tiasweb.info), IA is defined as "Integration of knowledge from different disciplines with the goal to contribute to understanding and solving complex societal problems, that arise from the interaction between humans and the environment, and to contribute in this way to establishing the foundation for sustainable development. Modelling and participatory processes should include stakeholder groups and the public at large." IA has been summarised by Jakeman and Letcher (2003) as:

- being problem-focussed, using an iterative, adaptive approach that links research to policy;

- possessing an interactive, transparent framework that enhances communication;

- being enriched by stakeholder involvement and dedicated to adoption;

- connecting complexities between the natural and human environment, recognising spatial dependencies, feedbacks and impediments; and

- attempting to recognise missing essential knowledge.

IA depends crucially on public participation, and on iteration of the development steps as more is learnt about the focus system. IA may be conceptualised as in Figure 1.

Integrated Scenario Modelling (ISM) is a core activity of many IA exercises. It involves constructing a model to approximate the system under study. The model allows simulation of the impact of changes in input drivers (scenarios), e.g. climate and human activities, on outputs (indicators) representing the states of the system. In this context, a state may be a sustainability target or an indicator that summarises an output, usually a simple function such as an average, a maximum or minimum level or a trend. The ISM component is at the centre of IA but must be extended in three ways, so that policy and management are linked to the controllable human activity inputs, stakeholders participate in all relevant parts of the process, and scientists and IA practitioners manage that process and its communication aspects. ISM outputs also play an essential part in multicriteria analysis, formal or informal. As summarised by Jakeman and Letcher (2003) ISM provides: 


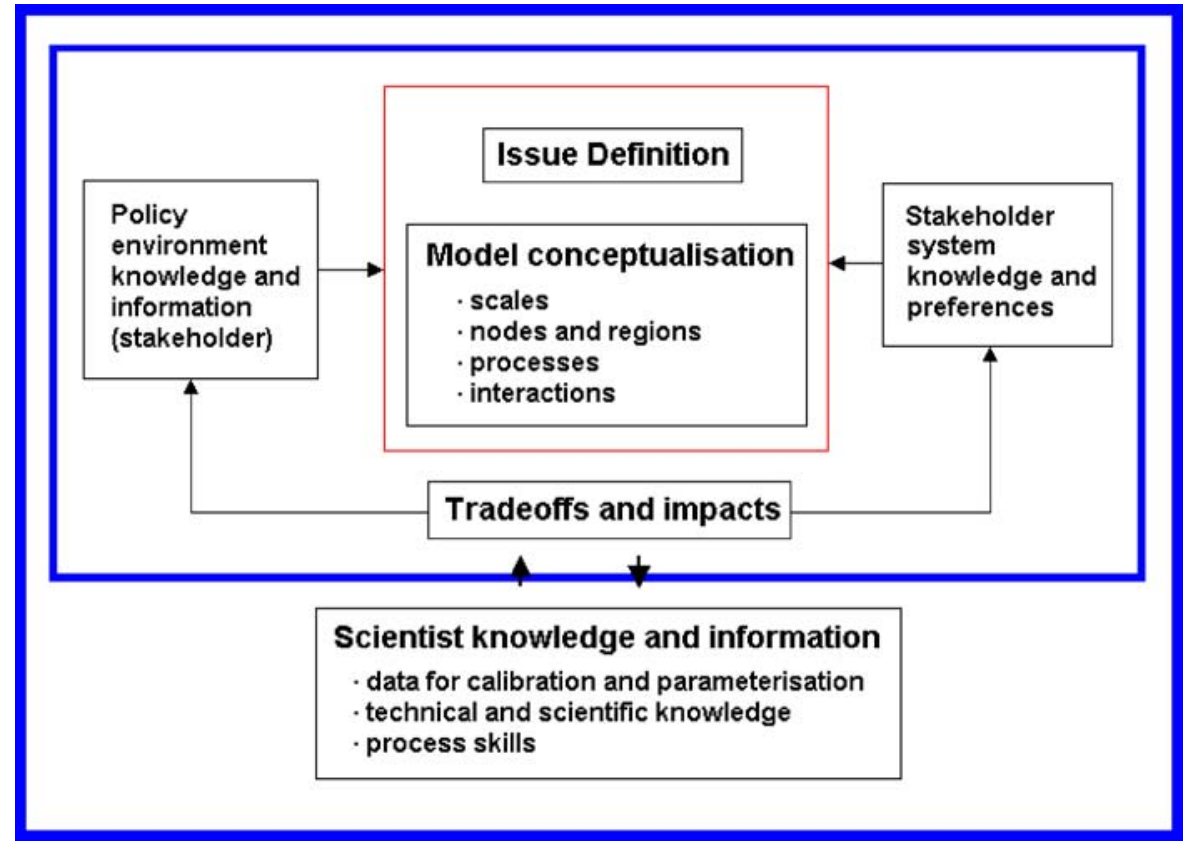

Fig. 1 Diagrammatic representation of integrated assessment

- a way of investigating and explaining tradeoffs;

- a readily accessible collection of models, methods and visualisation tools;

- a focus for integration across researchers and stakeholders;

- a training and education function;

- an exploratory aid capable of adoption and further development by stakeholders;

- a permanent summary of the project methods; and

- a means of making the management analysis transparent.

\section{A hierarchy of modelling problems}

The aspects of integration were characterised above from a management perspective. While still recognising that the model is for management and related purposes, this section focuses more on integration from a modelling perspective. In river basins as in other environmental systems, a hierarchy of problems require IA. The hierarchy is defined by several qualifiers: issues, scales, disciplines, and interest groups. One could begin with any of these, but the issues really determine the nature of the other qualifiers. Problems lower in the hierarchy have fewer issues of concern, scales to be considered, disciplines to invoke and interest groups to involve.

\subsection{Issues}

The issues that arise in river basins tend to be interrelated, especially when a problem has offsite or downstream effects, and often they are conflicting, so that one needs to integrate 
their assessment and management. The number of issues that must be considered in an IA tends to increase with scale.

\subsection{Scale}

Scale considerations enter into integration in three ways:

- the levels at which decisions require support;

- where and how model-output indicator functions should apply; and

- the temporal periods and spatial extents over which a model (and model componnents) must be run (the domain) and the time and space step (the discretisation).

Closely related to scale is the breakdown of a model into its subsystems. The breakdown depends on the management questions being asked and the nature of the data and other prior knowledge. In the course of IA, the real objectives are often found not to be broadly based, or can be simplified and remain useful for management, so that one may be able to ask questions in a modelling exercise that are less demanding than initially perceived (Jakeman, 1989).

\subsection{Disciplines and models}

At the simplest level, integration often refers merely to coupling models. More holistic use of the term 'integration' refers to a model or assessment that includes a mix of physical, social, economic, ecological or community perspectives. A mix of disciplinary approaches can lead to new insights into a management issue that could not be reached from a single viewpoint and, importantly, can lead to identification of critical gaps in disciplinary research and knowledge.

\subsection{Interest groups}

Environmental management and improving the sustainability of a system always involve trading impacts between different interest groups. Hence virtually all environmental management involves some level of conflict resolution and will require input from, and final adoption of the management solution by, a range of stakeholder groups. IA thus requires the integration of perspectives and input from a broad range of interest groups, some affected by the initial management issue and others affected by possible solutions.

\section{Reasons for and objectives of integrative modelling}

Broad reasons for integrative modelling include:

- improving system understanding;

- discovery, acquisition or elicitation of knowledge;

- social learning among interest groups;

- prediction or simulation;

- forecasting;

- management and decision-making; and

- discovering limitations, inconsistencies and gaps in data.

Springer 
These motives are not independent but should be considered by the IA modeller with regard to the appropriate framework, model type, available knowledge and data, and the desired outcomes.

The model developer (see Jakeman et al., 2006) should be clearly aware of the:

- questions and issues that the model is being developed to address;

- interest groups, including the clients or end-users for the model;

- outputs required;

- forcing variables or drivers;

- accuracy expected of the model;

- temporal and spatial scales;

- time frame for the completed model, e.g. for a decision;

- effort and resources available for modelling.

\section{State of integrative methods}

There are many different integration approaches, reviewed by Letcher and Jakeman (in press) and Letcher et al. (in press), but they share some aspects. Bayesian Networks, Agent-Based Models and Expert Systems, described below, might be considered as artificial intelligence or knowledge-based techniques. However their individual use as integrating frameworks warrants specific mention.

\subsection{System dynamics}

System dynamics (e.g., Deaton and Winebrake, 1999) is a modelling approach that investigates and manages complex feedback systems (e.g. aquatic food webs). Many authors consider it to be a philosophy of model development rather than an integrated approach. Nodes in the conceptual framework generally represent state variables, while the links or arrows between nodes represent functions transforming one state variable to the next. The conceptual frameworks for system dynamics models often contain feedback loops. These loops may be very complex, or may only represent perceived 'plausible' connections. Thus, system dynamics models are most commonly used to improve systems understanding and to compare simulation responses, rather than for decision-making and policy development. However, in theory the latter could be achieved.

\subsection{Bayesian networks}

Bayesian networks consist of a series of nodes and links that conceptualise a system, but feedback loops cannot be included in this approach. They are fundamentally a decisionmaking tool.

The nodes in the system are variables. The links are defined by conditional probability distributions (Borsuk et al., 2004), thus providing a measure of the certainty in the causal relationship between each pair of nodes. This integrated approach differs from others that use deterministic, rather than probabilistic, methods to determine the relationship among variables (Borsuk et al., 2004). The implicit ability to account for uncertainty means that Bayesian networks are able to make use of 'soft' sources of data, such as expert opinion, where observation data are not available (Pearl, 1988). 
Bayesian Decision Networks also include decision variables, which allow for management options to be implemented, and utility variables, which represent the benefit or cost of a particular decision (see for example Sadoddin et al., 2003).

\subsection{Metamodels}

Metamodels are essentially a simplified overall description of the processes within complex systems or more complex models. Data-mining techniques and regression are often used to develop metamodels.

Bouzaher et al. (1993) suggest that metamodels approximate, and aid in the interpretation of, simulation models. The mere size of the output from complex models can make them difficult to view and interpret. Metamodels can provide look-up tables or simpler functions to represent the information found by the more detailed models.

In integrated modelling, metamodels can be used to replace a complex model, or complex components of a model, completely. In the latter case, the metamodels can be coupled into an integrated system.

\subsection{Risk assessment approaches}

Risk assessment basically provides "a principled way of organising what we know about the world, particularly about its weak spots and creaky joints" (Jasanoff, 1993). Risk is defined as the probability of an outcome multiplied by the severity of its consequence, leading to the potential quantification of risk in a number of ways. This notion can also be extended to positive as well as adverse impacts and the characterisation of uncertainty.

Kammen and Hassenzahl (1999) present much of the central theory and methods including order-of-magnitude estimation, cause-effect calculations, exposure assessment, fault-tree analysis, and managing and estimating uncertainty.

\subsection{Coupled component models}

Coupling component models involves combining models from different disciplines to arrive at an integrated outcome. Conceptually each node in the framework represents a model of a particular issue. The links between models pass the generated data. The links maybe manually linked externally to the original models, or may be more tightly linked where the component models share inputs and outputs (e.g., Merritt et al., 2004; Letcher et al., 2004). Coupled component models are generally able to incorporate feedback loops.

Coupled component models can account for non-trivial temporal and spatial discretisation. This is particularly relevant in catchment and river basin management, where it is important to be able to isolate the impacts of upstream nodes and prior stream conditions. Two examples of this approach are presented in a later section.

\subsection{Agent-based models}

An agent- or actor-based model is essentially a type of coupled component model. It focuses on the interactions between agents (individuals) in a system (e.g., Brown et al., 2004), where 
agents adapt to changes to their environment. A system in which two or more agents exist at the same time, share resources and communicate with each other, is called a multi-agent system.

Agent-based models are efficient at identifying large-scale outcomes resulting from often simple, local interactions between individuals. For this reason, and because they tend to be hypothetical, agent-based models are usually applied in social and ecological science.

\subsection{Expert systems}

An expert system is a type of qualitative model where prior knowledge is encoded into a knowledge base and then logic is used to infer conclusions (Davis, 1995). The knowledge base determines the success of the system (Forsyth, 1984). Given a problem, the expert system simulates the problem-solving task(s) (Kidd, 1987). The conceptual diagram for an expert system refers to questions about the nature of the system directed at the user. The response to these questions then dictates the route down which the procedure looks for a solution.

\section{Other artificial intelligence methods}

Innovation in methods that deal better with uncertain and/or qualitative knowledge is essential for progress in making sustainability assessments. Artificial intelligence techniques have a long history of promise but do not seem to have delivered yet in this context. They are potentially particularly useful for knowledge acquisition; see, for example, the short discussion on data-mining techniques below. According to Sell (1985), there are four primary sources of knowledge: literature, human specialists, existing models and examples. Schmoldt (1998) initiates attempts to organise the different methods of knowledge acquisition by specifying a conceptual approach known as linguistic-based knowledge analysis to develop lexicons, syntax and semantics for a domain. While many natural-resource domains present unique sets of problems in acquiring existing expertise, he argues that there are enough commonalities to be exploited by sharing knowledge acquisition experiences.

Likewise, the emerging field of environmental informatics has much promise. It combines artificial intelligence, GIS, software frameworks for modelling and linking models, and user interfaces, but effective capture of knowledge has lagged far behind software implementation. As with artificial intelligence, environmental informatics also needs urgently to be complemented by user-driven IA providing priorities, structure and efficiency. Without such application-oriented discipline, environmental informatics runs the risk of becoming capable of doing a wide range of things, all badly.

A taxonomy of knowledge-acquisition categories (and particular techniques within each category), as provided by Schmoldt (1998), includes: unstructured and structured interviews (using free association, analytic hierarchy processes, psychological scaling, sorting and knowledge diagramming); questionnaires; automated tools; problem solving (familiar cases, limited information cases, tough cases); machine learning (induction from examples, neural networks, genetic algorithms); and protocol extraction (goal decomposition, forward scenario simulation, verbal protocol, retrospective protocol).

Data mining is a discipline that covers some of these techniques and merits brief individual mention for illustrative purposes. It also has enormous potential for knowledge extraction in both socioeconomic and biophysical applications. 


\subsection{Data mining}

Data mining can be defined in many different ways. Following Spate et al. (2003) it can be the discovery of interesting, comprehensible and previously unknown rules, trends or characteristics from data. While much of the knowledge sought with data-mining techniques could, in principle, be extracted by detailed and rigorous visual or statistical investigation, that may involve impracticably large time and computation costs. This is especially so when prior knowledge is not good enough to point to where to look in large data sets. The quite large number of techniques and tools available includes clustering, classification, association rule extraction, dominant mode analysis and time series similarity measures.

\section{Opportunities and challenges}

\subsection{Incorporating key considerations in integrated modelling}

Some of the modelling considerations listed below should be addressed more routinely in the management of natural resources (Jakeman and Letcher, 2003). Few examples in the literature demonstrate systematic attention to them.

- Climate variability and episodes

- Modelling process complexity

- Beyond business-as-usual scenarios

- Modelling long leads and lags

- Narrowing modelling objectives

- Assessment of model sensitivity and uncertainty

- Error accumulation

- System representation

\subsection{Quality assurance for credible models}

Quality assurance refers to the standards and protocols for model and data reporting and distribution (see stars.net.au). It is contended that this type of regulation is required to enhance the credibility and ultimate utility of models. Uniform reporting on the limitations and assumptions in models and associated input data would empower model users and decision makers to use models appropriately.

It is suggested that model credibility can be enhanced by:

- a serious two-way modeller-stakeholder dialogue;

- appropriately rigorous model evaluation tests;

- sensitivity and uncertainty assessments; and

- peer reviews of models at their various stages of development.

Refsgaard et al. (2005) classify guidelines "according to how much focus is put on the dialogue between the modeller and the water manager as: (Type 1) Internal technical guidelines developed and used internally by the modeller's organisation; (Type 2) Public technical guidelines developed in a public consensus-building process; and (Type 3) Public interactive guidelines developed as public guidelines to promote and regulate the interaction between the modeller and the water manager throughout the modelling process." 


\subsection{Recognising broad objectives}

It should be remembered that the broad objective of modelling is to increase understanding of the directions and magnitudes of change under different management options. The ability to differentiate the relative impacts from change compared to the 'do nothing' scenario is all that really can be supported in the current modelling environment. Treating simulation outputs as accurate predictions is unrealistic. To assist in policy implementation and decision making, the ability to ascertain at least a qualitative measure (high, medium, or low) of certainty in the outputs is required. Ideally, predictions would be produced with a quantitative confidence level, but in most situations this is impracticable at present. Currently, methods for quantifying uncertainties have severe limitations. Similar comments go for sensitivities of model outputs with respect to variation in parameters of the model itself or its drivers and boundary conditions. Norton et al. (2003) and Jakeman and Letcher (2003) discuss new research required to address this deficiency.

\subsection{Participatory modelling}

Public participation can be defined as direct involvement by the public in decision-making (Mostert, 2006). There are several reasons for organizing public participation including the possibility of:

- more informed and creative decision making;

- greater public acceptance and ownership of the decisions;

- more open and integrated government;

- enhancing democracy; and

- social learning, the ultimate objective, to manage issues.

According to Mostert (2006), there are three principles of social learning:

- reflection on goals - participants are encouraged to reflect on why they want to attain the goals they are seeking, as there may be better ways to achieve what they want;

- reciprocity - participants must realise that they are interdependent, and should consider others in their actions and information exchange; and

- respect for diversity - participants should acknowledge that other participants may have different cultures, backgrounds and interests.

He also notes that public participants must be open and honest about their opinions, issues and concerns, as defensive behaviour will hamper the coordinated approach. The process for including public participation in decision making must be well organised and sensitive to cultural differences in order to maximise the potential benefits. This requires leadership and facilitation to ensure that all participants are given opportunity to speak freely. To further the benefits, public opinion should also be considered when designing relevant scientific research and in the presentation and dissemination of results.

IA can be a useful mechanism for public participation. For example, public consultation during the development of a decision-making model means that different perceptions can be acknowledged and accounted for within the model structure. The final product is then more likely to provide output useful to the public. Conflict that arises during discussion in model development may identify areas of disagreement or knowledge gaps, thus indicating that it should be a priority in the project to collect data and improve understanding of that issue. Such conflict resolution is usually received positively by most stakeholders, as they feel that 
their concerns were listened to and addressed by the process. Hare et al. (2003) present a recent comparison of different participatory processes.

The output that IA produces to assist in decision-making should be dynamic, so that the tools evolve and remain usable as the knowledge, systems understanding, goals, and conflicts of the participants develop, or as the landscape responds and changes. In this way, tools should account for short-term learning and goals, in the light of long-term visions of sustainability.

\subsection{Adaptive management}

Adaptive management (Holling, 1978) and active adaptive management (e.g., Allan and Curtis, 2003) are principles to improve environmental management through learning. They can be used to develop management-revision principles, experiment design, outcome indicators and monitoring practices. Designed assessment and evaluation of current and past experiments can identify past successes and failures, and the knowledge gaps.

Adaptive management implies feedback from monitored outcomes to revised actions, as in control engineering. Some essential issues confronting adaptive management can be identified by examining what factors are crucial in employing designed feedback in control engineering:

- determining what behaviour dominates the system's response under control, and specifying simple requirements for that response;

- observability (ability to see, through monitored outputs, the behaviour which is to be controlled) and controllability (ability to influence, through available inputs, the behaviour which is to be controlled);

- comparison between measured and desired output to determine error and generate corrective control action;

- "actuator constraints", i.e. limitations on the action that can be applied to the system; and

- consideration of robustness of control-system performance over a range of uncertainty in the model and the system's environment.

Such ideas are fundamental in design of feedback systems but it is surprising how little discussion their relevance to environmental modelling and management has had.

\subsection{Targeting disciplinary gaps}

To utilise fully the benefits offered by IA, there needs to be greater integration of all disciplinary resources. The following case studies represent just a tiny fraction of potential examples. The social sciences, for instance, can offer insight and information into the decisionmaking and adoption processes previously ignored in many scenario-based models. In particular, social survey data, linking information about decision-making and adoption to the biophysical and socioeconomic characteristics of farmers, industries or households, is crucial to developing more sophisticated ISM and other policy analyses. Public participation in model development and testing is one way to account for the social considerations in a particular system (e.g., Haslam et al., 2003).

Artificial intelligence techniques offer an interesting and useful alternative to theorybased models of biophysical and socioeconomic processes. Theory-based models developed to maximise profit, for example, are difficult to validate. However, where 'soft data' (i.e. interview and survey results) are used to govern decisions, the model performance and outcomes can be easily tested through further public participation and validation. Comparison of the output from the two approaches (theoretical and artificial intelligence) could indicate 
whether the greater complexity in the theoretical approach is warranted, and how robust current techniques are.

\section{Australian case studies}

In this section we present three examples of IAM projects in Australia to illustrate the potential positive impacts of the IA process and the modelling therein. The key components of the IA for each are summarised in Table 1.

\subsection{Water allocation project (Namoi and Gwydir valleys, NSW)}

The sustainability of groundwater stores and streamflow in some of Australia's agricultural regions is under pressure from an increase in the number of irrigators and their irrigation requirements. In this project a decision-support system (Letcher et al., 2004; Letcher and Jakeman, 2003) was developed. This DSS is accessible to industry representatives and state government agency staff, for considering the impact of water allocation policies on water users and the environments of the Namoi $\left(40,000 \mathrm{~km}^{2}\right)$ and Gwydir $\left(30,000 \mathrm{~km}^{2}\right)$ River basins in NSW.

To achieve these outcomes the model includes the ability to differentiate spatially and temporally between:

Table 1 Comparison of integrated assessment approaches for three Australian case studies

\begin{tabular}{|c|c|c|c|}
\hline & Namoi/gwydir & Ben chifley & Coastal lakes \\
\hline Spatial scale $\left(\mathrm{km}^{2}\right)$ & 40000 & 985 & 23 to 1660 \\
\hline $\begin{array}{l}\text { Dominant land use activ- } \\
\text { ity }\end{array}$ & $\begin{array}{l}\text { - Rotational based } \\
\text { irrigated and dryland } \\
\text { options }\end{array}$ & $\begin{array}{l}\text { - Sheep and beef cattle } \\
\text { grazing, forestry }\end{array}$ & $\begin{array}{l}\text { - Urban settlement, } \\
\text { - Nature reserves/national } \\
\text { park, recreational water } \\
\text { sports, various } \\
\text { agricultures depending } \\
\text { on the lake }\end{array}$ \\
\hline $\begin{array}{l}\text { Key water management } \\
\text { concern }\end{array}$ & $\begin{array}{l}\text { - Water allocation as } \\
\text { it relates to agricultural } \\
\text { production and envi- } \\
\text { ronmental flows }\end{array}$ & $\begin{array}{l}\text { - Land management } \\
\text { impact on water qual- } \\
\text { ity for recreation and } \\
\text { town water supply }\end{array}$ & $\begin{array}{l}\text { - Land and water manage- } \\
\text { ment impacts on coastal } \\
\text { lake health for social, eco- } \\
\text { nomic and environmental } \\
\text { use }\end{array}$ \\
\hline $\begin{array}{l}\text { Modelling approach } \\
\text { Management options/ } \\
\text { policies that can be } \\
\text { explored with the model }\end{array}$ & $\begin{array}{l}\text { - Coupled } \\
\text { - Off-allocation } \\
\text { - Groundwater alloca- } \\
\text { tion reductions } \\
\text { - Sleeper license acti- } \\
\text { vation, } \\
\text { - Volumetric conver- } \\
\text { sion } \\
\text { - Daily flow extraction } \\
\text { rules } \\
\text { - Environmental flows }\end{array}$ & $\begin{array}{l}\text { - Coupled } \\
\text { - Riparian revegeta- } \\
\text { tion or clearing, } \\
\text { - Establishment of } \\
\text { buffer strips with stock } \\
\text { exclusion } \\
\text { - Engineering river or } \\
\text { gully stabilisation } \\
\text { - Land use change } \\
\text { - Change in emission } \\
\text { from point sources }\end{array}$ & $\begin{array}{l}\text { - BDN } \\
\text { - Lake entrance openings } \\
\text { - Location of urban devel- } \\
\text { opment } \\
\text { - Urban development con- } \\
\text { trols (stormwater and sew- } \\
\text { erage management, flood } \\
\text { mitigation) } \\
\text { - Agricultural manage- } \\
\text { ment practices (fertiliser } \\
\text { application, buffer strip } \\
\text { controls) } \\
\text { - Riparian management }\end{array}$ \\
\hline
\end{tabular}


- changes in regulated, unregulated, groundwater and supplementary allocations or entitlements;

- changes in "commence" and "cease to pump" thresholds (in river flow) for unregulated and supplementary water, as well as changes in daily extraction limits, including the option of multiple pumping regimes throughout the year;

- changes in carryover rules for regulated water, unregulated water and groundwater, including the option of no carryover;

- changes in the cost of water for different systems; and

- the influence of climate on the impact of these changes.

These issues were identified in consultation with stakeholder groups (see Letcher et al., 2003).

The model accuracy is sufficient for a decision-based tool, but the complexity is kept as low as possible so it can be used in an integrative framework. The decision support system enables the evaluation of tradeoffs between socioeconomic indicators and environmental flows. More specifically, it represents impacts and effects from:

- allocation of groundwater;

- change in the number of irrigation licences;

- environmental flow of the streams; and

- the capacity of farmers to adjust their practices.

Stream health (flow) indicators, agricultural production and how these vary across the basins are used to gauge basin health.

Spatial variation is addressed by dividing the basin into regions of similar groundwater policy, surface water policy and production type. These regions for the Namoi river basin are shown in Figure 2a. The integration of socioeconomic and environmental factors is achieved using a coupled model approach. Hydrological, policy, economic and extraction submodels are integrated to address the given concern. The daily streamflow into a region under particular climatic conditions is simulated and fed into the policy model. This gives the total volume of water available for irrigation in each month, which is used in the economic model to determine farmers' decisions on water management, irrigation practices and crop planting (areas and types). The total water extracted from the stream is then calculated and the remaining water flow is available for input into the downstream region.

The results presented in Figure 2 show the strength of this decision support tool in integrating economic and environmental factors spatially to assist in decision-making for the Namoi river basin. The tool was run given various percentages of sleeper (currently unused) licence activation throughout the basin. Figure $2 b$ shows that the regions in the top of the basin (A, B, D and $\mathrm{G}$ ) receive a continuous increase in their profit (to up to 3.0\%) with increased water allocation, but in the lower basin (regions $\mathrm{M}, \mathrm{N}$ and $\mathrm{O}$ ), when over $40 \%$ of sleeper licences have been activated some regions begin to decrease in profit. Thus the expansion of irrigation in the upper basin comes at a cost to the lower basin users. Figure $2 \mathrm{c}$ shows the impact on streamflow under the same scenarios presented in Figure $2 \mathrm{~b}$. There is up to $15 \%$ reduction in non-zero median flows in the upper basin (regions B, D, E, F, G and $\mathrm{H})$, the greatest reductions being created when the percentage allocation of sleeper licences exceeds $55 \%$. There is also up to $5 \%$ reduction in non-zero median flows in some regions in the lower basin (J, K and L) when licence activation exceeds $60 \%$. Thus for a comparatively small increase in profit in the upper basin, the profits in some regions of the lower basin will decrease, and there will be a strong reduction in non-zero median streamflow in the upper 

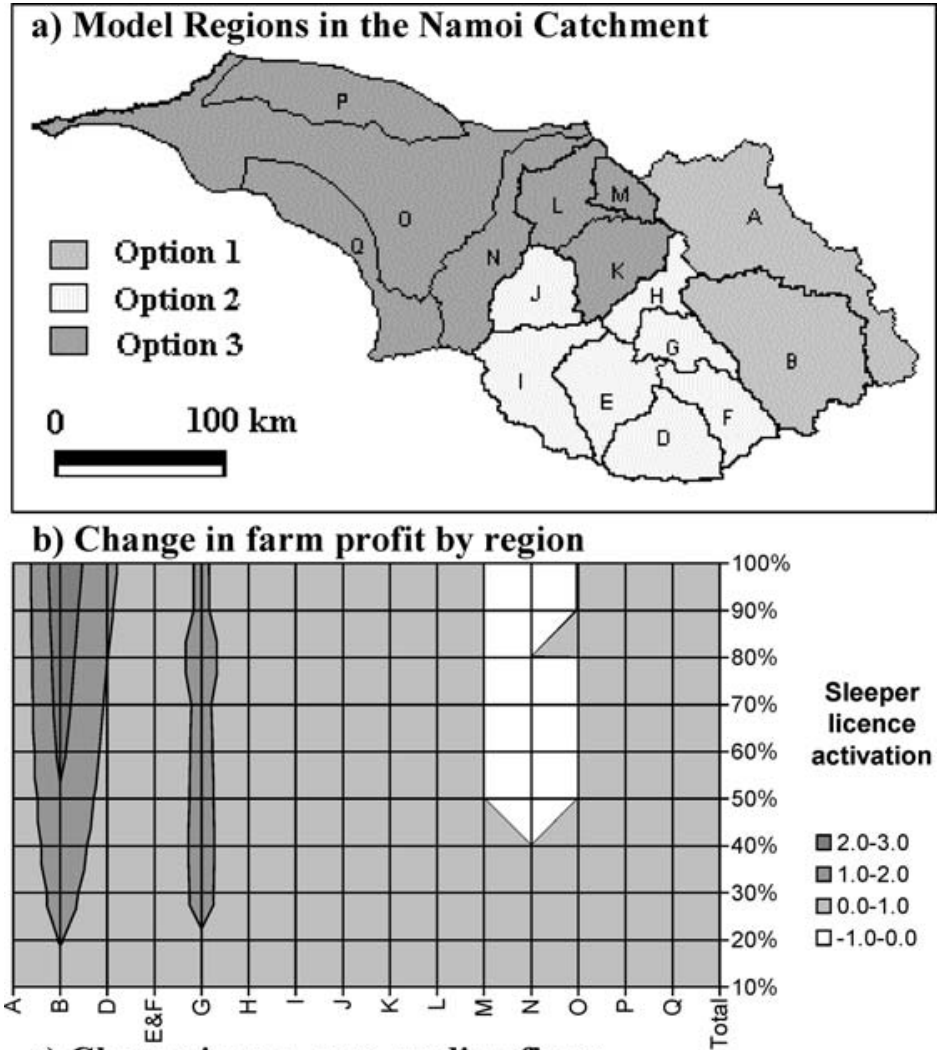

c) Change in non-zero median flows

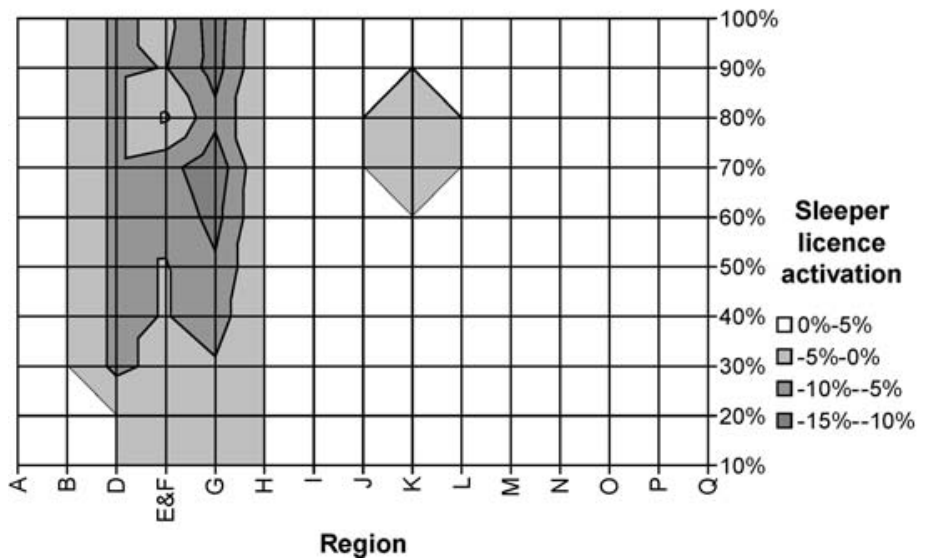

Fig. 2 Example of results from the Namoi decision support tool

basin. These results effectively show the implications of policy changes for the economic and environmental quality of the basin.

This water allocation project has been a collaborative undertaking with input from NSW Agriculture, the NSW Department of Infrastructure, Planning and Natural Resources, the Australian Cotton Cooperative Research Centre and irrigator groups. Stakeholder participa- 
tion was an important component of the model development process. Workshops were held to identify the controls on water use and drivers for on-farm water use, and to refine and test the model.

Outcomes from the project include:

- delivery of decision-support tools for the Namoi and Gwydir river basins, accessible through user-friendly interfaces;

- training workshops for industry, state government agency staff and other community representatives; and

- feedback from industry, state government agency staff and other community members to ensure that the models are as accurate as possible, and that the interface is designed to meet the needs of these groups.

Many obstacles were faced in the course of the project, despite the successful outcomes presented above. Because of the scale of the Namoi and Gwydir catchments, there is an inherent diversity of interest in the use of the waterways, with a large number of stakeholders involved. Consequently, the consultation had to be targeted, but is still believed to be representative. However, the resulting model may be biased by the level and type of consultation. There is also considerable political and economic pressure within the Namoi and Gwydir catchments, leading to substantial criticism of water reforms and estimates of impact as well as conflicts over water use. This type of pressure is likely to affect any participatory process undertaken in these catchments. There are also large differences in the views of individual farmers and government agency representatives, so community participation relying on representatives from the groups had to involve mechanisms for revealing and incorporating these different opinions.

Initially there was an unwillingness by various stakeholder groups to commit early on to supporting the project. All groups essentially required clarification that one or more other groups had agreed to support the project before they would agree to be involved. This tended to slow the initial model development, through lack of active community participation from some groups in the early stages. However, it can also be viewed as an advantage because it meant that no particular stakeholder group 'took control' of the whole project, so there was less room for bias in the early, perhaps more vulnerable, stages of the project.

Once stakeholder participation had increased, there was evidence of a trend of overcommitment by individuals, a situation likely to occur in many catchments throughout Australia. The problem is that people who have an interest in participating in such projects, and are active and respected within the catchment, often commit extensive time and resources to the project. This of course is desirable to a point. But if one key person commits to too many projects, then they can become over-committed and spend too much time in meetings and workshops. This can be at the expense of keeping in touch with the catchment and the community they represent, and potentially may damage their own financial and personal interests.

\subsection{Pollutant management project (Ben Chifley Dam Basin, NSW)}

A concern of many water managers is elevated nutrient levels in water supply reservoirs. Increases in nutrient levels may increase the likelihood of algal blooms. The objective of this project was to develop a modelling system to assist in the management of diffuse pollution (nutrients and sediment) inputs into reservoirs. The project was focused in the Ben Chifley Dam catchment, a 1,000 $\mathrm{km}^{2}$ basin in the central tablelands of NSW (Newham et al., 2004a). 
In order to address this problem, the IA model had to account for the key controlling factors on dam water quality (climate and associated hydrologic factors, topography, land use and management of the riparian zone) as well as climate variability and the socioeconomic costs of remediation and adoption. It also had to be sensitive to spatial and temporal patterns in order to target remediation works.

Again a coupled model approach was selected, which included hydrologic, sediment, nutrient and economic sub-models. The resultant IA model is called CatchMODS. In CatchMODS, the basin is divided into stream reach and sub-basin elements. Various riparian management and land use changes can be applied to each. Figure 3a shows the sub-basins and stream reaches used for the Ben Chifley Dam basin, and the larger management subbasins. Given the defined land use and riparian conditions, the hydrologic model is run for the highest reach of the basin. The mean annual flow, mean annual base flow, median overbank discharge, and bankfull stream flow are calculated and used as input into the sediment and nutrient sub-models. The resultant sediment and nutrient outflows are routed into the next downstream reach.

Outputs from the modelling system include mean annual estimates of the fluxes of total suspended sediment, total nitrogen, total phosphorous and stream flow, together with the fixed and ongoing costs and change in agricultural gross margin associated with the implementation of each management scenario. Figure $3 \mathrm{~b}$ shows the spatial variation in suspended sediment export for the basin, given the current land use and management conditions. Outputs such as this allow model users to identify areas where remediation to control inputs should be targeted. Figure 4 shows the estimated costs and potential sediment reduction of channel remediation options for the Lower Campbells River sub-catchment. Such outputs enable model users to explore the tradeoffs between various remediation options for a single sub-catchment and also to compare the effectiveness of remediation between catchments.

Community consultation and participatory activities were incorporated into the process of model development. Input was sought from a range of interest groups, including State and Local government, individual landholders and community-based natural resource management organisations. The development of environmental models provided a very useful focus for communication activities between scientists, basin managers and the broader basin community. In this way, the impact of policy and management strategies could be effectively evaluated, and stakeholders and researchers engaged in a two-way interaction to facilitate the adoption of practices to improve basin sustainability (Newham et al., 2004b). A weakness of the participation activities in the case study was that the focus of the delivery of the IA model was a single organisation, the Ben Chifley Catchment Steering Committee. This resulted in rapid progress in development of the model but presented difficulties in the broader adoption of the results of the research following a significant restructure of natural resource management agencies in the catchment.

\subsection{Sustainability assessment of coastal lakes (pilot study for eight lakes in NSW)}

Coastal lakes are ecosystems of significant value, generating ecological, social and economic benefits enjoyed by a large proportion of the NSW community. They are under increasing pressure from encroaching urban development and intensification of agricultural practices, which threaten the very qualities that make the lakes so inviting. This project aimed to develop a tool to assist in planning and management of the basins of eight coastal lakes in NSW, in order to promote the sustainability of their function (Newham et al., 2004c). A separate tool is developed for each lake, following the process outlined below. 
a) Management subcatchments within the Ben Chifley Dam catchment

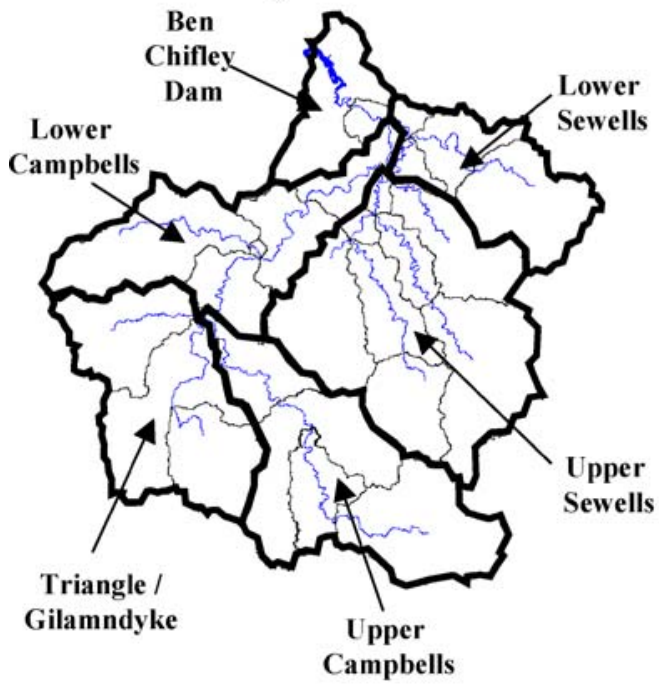

b) Suspended sediment export for each subcatchment

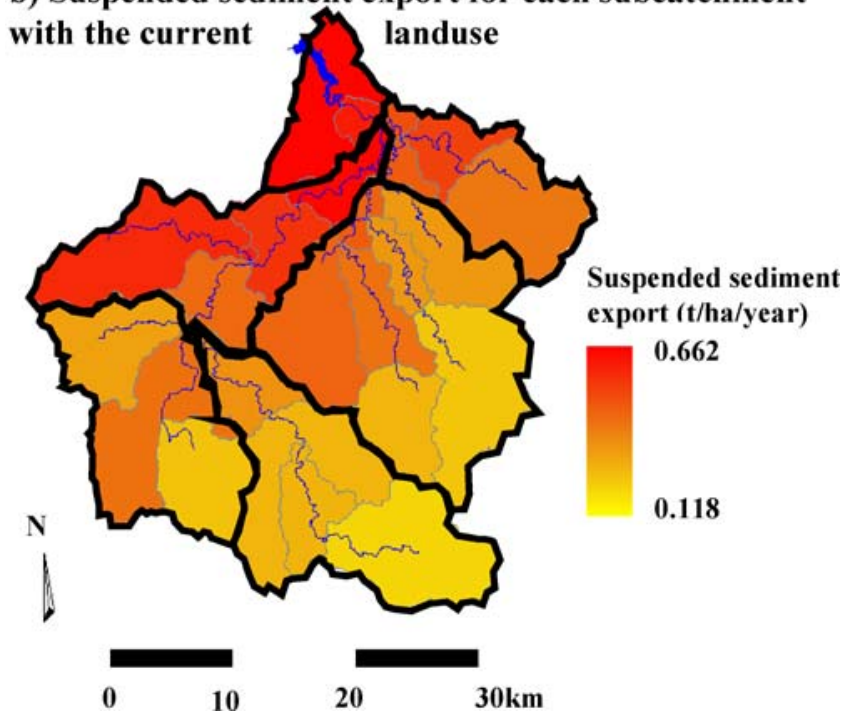

Fig. 3 Example of results from the CatchMODS tool for the Ben Chifley Dam basin

To develop a useful tool, the key constraints, issues and drivers impacting on each lake's health had to be identified by reading management plans and literature and through workshops with stakeholders. This identified many social, environmental and economic issues, which operate at various spatial and temporal scales. To account for this complexity and the time constraints on the project, a Bayesian decision network modelling approach was chosen.

Local stakeholders, including government agencies, shire council and farmers for each lake, were invited to assist in the development of potential management scenarios, such as Springer 


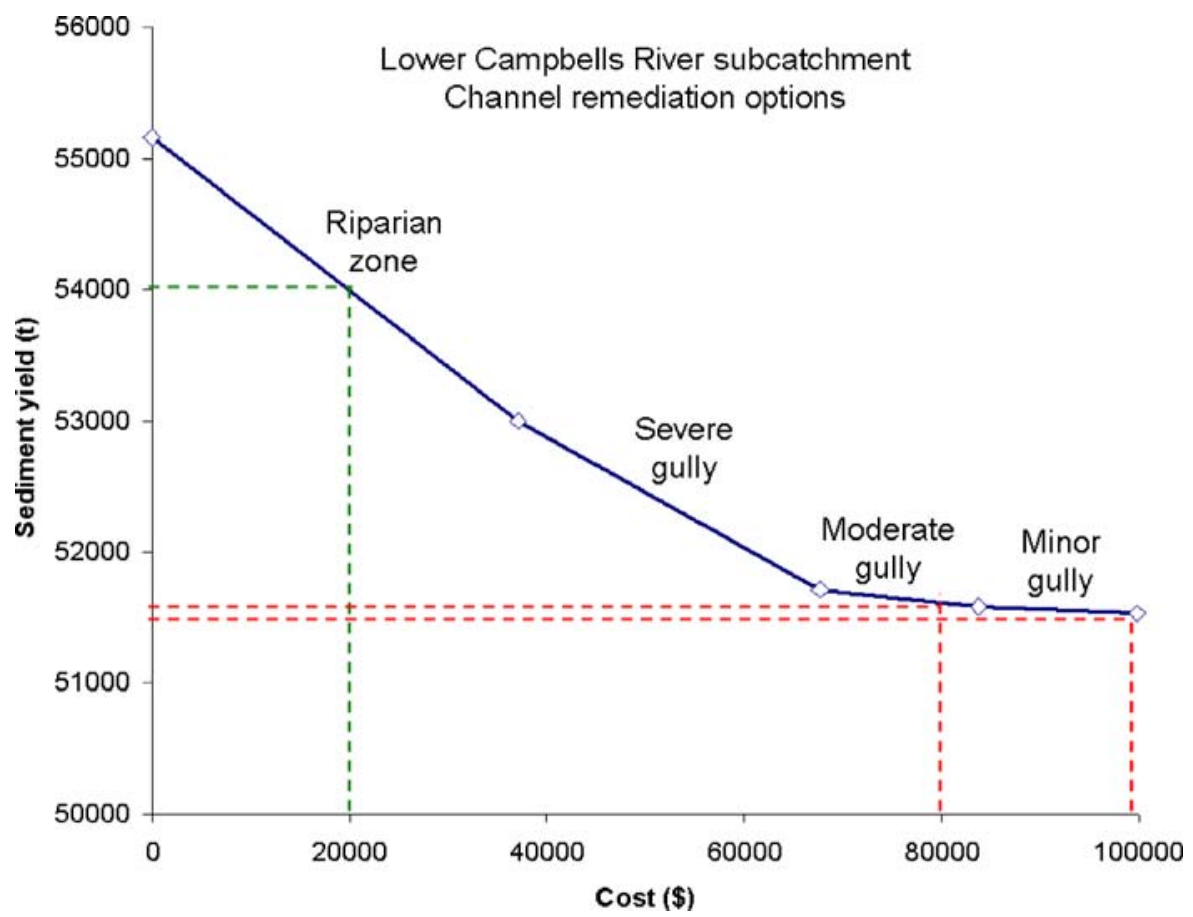

Fig. 4 An example output of CatchMODS from the Ben Chifley Dam subcatchment, of the costs and potential sediment reduction of channel remediation options

urban development, stormwater management, riparian zone management, and agricultural practices, which impact on lake health. They were also asked to identify the important economic, social and environmental costs/benefits that they deem important, (e.g. threatened species, recreation, tourism, oyster production) which may be impacted upon by the lake health. Given stakeholder knowledge and sought expert opinion, the key environmental indicators between the identified management scenarios and costs/benefits were linked to form the Bayesian decision network framework. The framework for one of the lakes, Cudgen Lake, is shown in Figure 5. This approach clearly illustrates the interrelatedness of the issues and values within the catchment. The BDN approach allows each variable within the model to be represented at an independent scale, so for each the scale can be selected that is most representative of that process, value or level required for decision support. The probability densities for each link were filled using model simulation, analysis of existing data and expert knowledge.

The key output from this modelling approach is an easy-to-use model interface that can assist council planners and other decision makers in making trade-offs between social, economic and environmental factors that affect their lake's health. The framework developed in this original pilot study can be easily altered. Thus if more detailed data become available to compile probability densities, or additional issues are identified that should be considered in the management of the lakes, the model can be updated. This dynamic approach allows users to iterate easily and further develop the tool so it can always meet their current needs.

The key challenge for this project was the time constraint on project completion, approximately one year. This limited the time that could be spent in building a relationship 


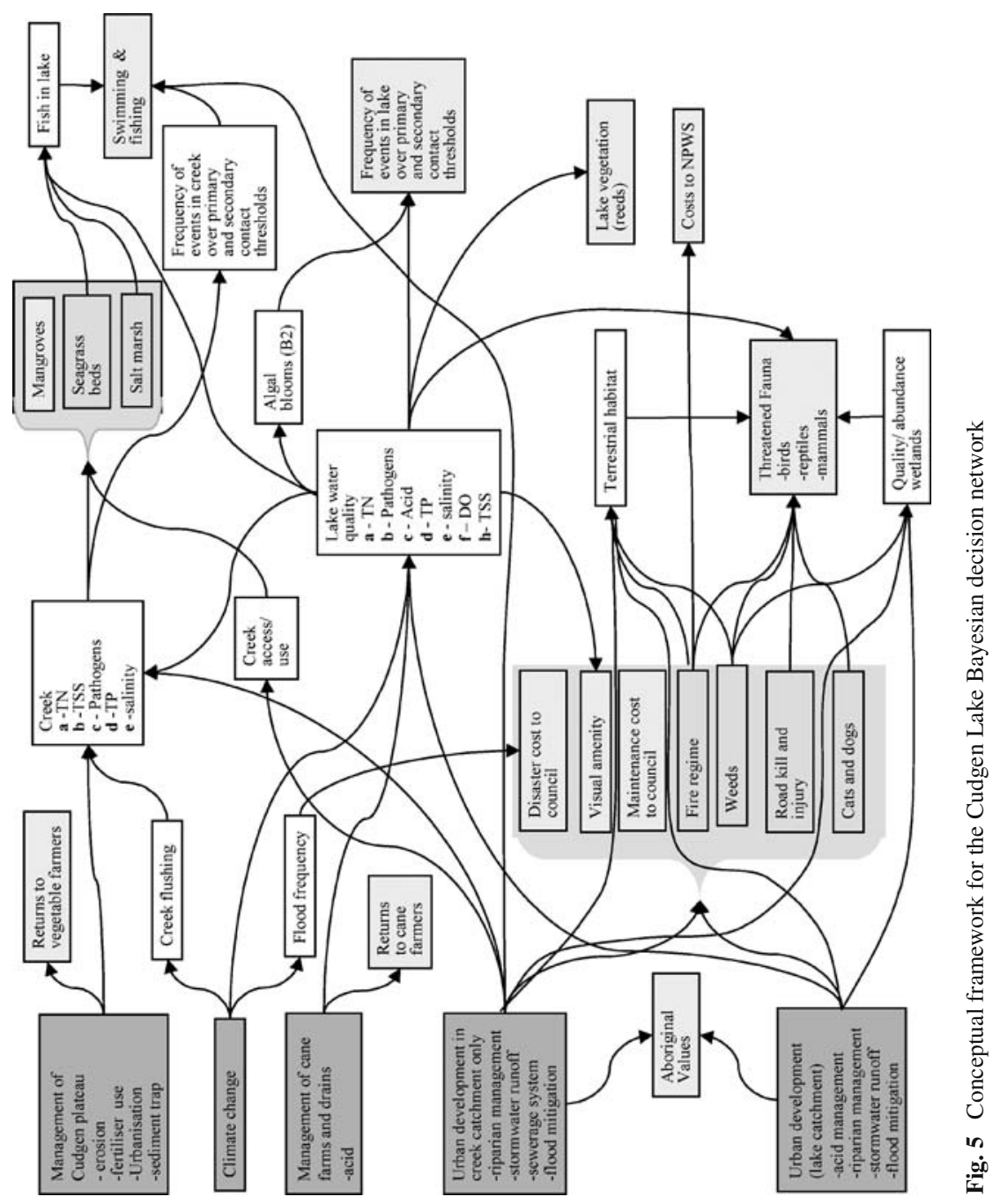

黛Springer 
and confidence with the stakeholders. Nonetheless, almost all community stakeholders were enthusiastic about the project and provided as much input as they could in the allocated time.

Another key constraint for the BDN approach is the inability or unwillingness of experts in the various disciplines to provide model input at a general level appropriate for the model. Many experts appeared to concentrate so much on the complexity of the system that they were unable to agree on and make generalised statements about the impact upon that system of given input changes. For example someone with detailed knowledge of the dynamics of fish populations by species might be unable to group fish populations or identify key indicator species in order to answer general questions on the change in fish populations given changes in water quality, fish catch and urban development. This inability restricts integrative models as presented here to existing, narrowly disciplinary research on the social, ecological and economic values, biasing the model and drastically reducing the potential of the integrative approach.

\section{The general problems and impacts}

IA or ISM projects may assist in directly achieving sustainability, but it is important to recognise that the most useful outcome may be in the learning experience of researchers, IA practitioners and stakeholder groups. Providing different stakeholders groups with opportunity to state their concerns and thoughts on management in an open unbiased forum can lead to improved understanding of the integrated nature of sustainability by participants in the project, an outcome worth achieving. However, it is important to note that the process is open to manipulation by the stakeholders consulted. People who are keen to participate may do so to 'control' the output and results. Their views will be heard and considered, while those who do not 'scream the loudest' may not be adequately represented. Some of the benefits of stakeholder participation in the development of decision management tools are evident from the case studies discussed above. However, successful participatory research requires substantial commitment of time and goodwill from the individuals and organisational groups involved. Even if these are attained in the establishment stages of a project, stakeholder participation can suffer if the key people driving the project for the community, or driving groups within the community, leave the catchment or change jobs.

Issues of commitment come from both sides. For example, people can be reluctant to provide input to a project because the information that they provide is then taken from their control and there is a fear that it might be used to their detriment. One possible means to avoid this, as was done in the Namoi/Gywdir project, is to introduce stakeholders early in the process and involve them in model development, the assumptions used and the input data. Thus they have a large input into model development and even if the model results are not favourable for them, they are less likely to criticise the model. It is important not to show preliminary results, as this can undermine stakeholder confidence in the researchers. There is a danger that select people may become too committed to the community consultation process and potentially become removed from the community that they are meant to represent. An intermediate level of commitment is desirable but difficult to achieve.

Valuable outputs from the process (e.g. increased knowledge of the environmental systems; enlightened or creative management suggestions; increased acceptance and uptake of models to support management decision making; improved access to and interpretation of existing data; and enhanced cross-agency collaboration) are often not readily measurable outcomes. This makes it difficult to attract funding, as funding bodies tend to target projects that deliver visible results. 
Adoption can be used to gauge the success of a tool or model. However, what is the definition of adoption? Adoption of integrative tools is multifaceted, dynamic and evolves over time. Typically adoption is considered to be the use of a tool or software following completion. However, adoption is also achieved if stakeholders embrace and employ the concepts identified in a tool through increased understanding of the interactions within a system. It is often difficult to gauge the extent of this type of adoption.

It is suggested that for successful participatory activities in support of integrated basin modelling:

- consultation activities are included as a feature of the entire project cycle;

- multiple mechanisms are developed for groups and individuals to be involved;

- input is invited from a broad range of organisations and individuals, and

- sufficiently long time scales are made available to develop trust.

If a long time scale is not possible, it is recommended that at least one key contact is made in the study basin to help in accelerating the development of relationships between the local stakeholders and the research party.

The Namoi Basin research over the last four years involved IA of water allocation options. The strong stakeholder focus in all stages of model development and testing has led to strong industry and government support for future work in the Namoi and Gwydir Basin. Pursuing model development with a clear and open process has resulted in stakeholders making and continuing to make valuable contributions and improvements to the model development and function. This has led to a sense of empowerment and ownership of the model by the stakeholders. With their increased understanding of the system that they live in or work with, it is hoped that they will use the model as a tool to assist decision-making into the future and not misuse the results for personal gain.

There were difficulties in the Namoi and Gywdir study because of government restructuring, resulting in some participants changing employment roles or leaving the catchment. This changed the key catchment 'drivers', setting back the participatory process.

The Ben Chifley Dam study developed a software tool based on science as accepted by stakeholders. The strong stakeholder participation and the simple nature of the model tool has generated strong interest from the regulators to make the software available for basin managers right across the state of New South Wales. This project too ran into difficulty in adoption because initial consultation was focused on one particular catchment group. This group was decommissioned because of a government restructure, making it difficult to push the adoption and continuation of the project and approach within the catchment.

IA exercises promote engagement by stakeholders and systems thinking, which can only assist people in appreciating one another's perspectives. This is particularly evident in the coastal lake project. The Bayesian decision network approach enables stakeholders and decision makers to see, with a glance at the framework, the variables which their management decisions may impact upon. After running the model they are able to identify the likely impacts of that management decision. This increase in awareness of the impacts on other lake users is likely to promote greater synthesis in lake management within the community. Again there are difficulties due to the restructuring of the lead agency. Another major obstacle is having experts commit to presenting their expert opinion on a particular topic in a simple manner. Often, experts with disciplinary focus pay too much attention to the complexities inherent in the system, and are unwilling to make the generalisations necessary for integrated studies. 
Consequently, it is difficult to find people who have a good knowledge of how the system works but who are still happy to make an expert judgement on the general changes expected.

In these three projects the ISM tools and exercises have also provided information and insights that would not have otherwise been possible. For example, in the Namoi, the initial stakeholder focus was on allocation of water in unregulated parts of the basin, but through ISM their concern additionally focused on other access rules for irrigation water. It also clarified that the timing of impacts was important, not merely the average impact. The cumulative impact after a series of dry years manifested itself as a major concern. The estimation of the impacts and the possible tradeoffs would not have been possible without models that in this case are predominantly quantitative.

All of the IA projects in which we have participated have reinforced the conviction that software development must be undertaken with a clear picture of the target audience, specific issues and uses. In none of the cases discussed here do the tools developed aim to provide recommendations to the land managers or to provide an "optimal" answer. That would inevitably require the model developer to determine what the optimal response is, tending to alienate stakeholders and thus hinder adoption. Instead these tools present potential outcomes or trade-offs that should be considered in managing the system. Thus while a sophisticated, object-oriented software platform may be both useful and desirable in some circumstances, in other cases a spreadsheet-based model may be more useful for these purposes. Different software products aimed at different audiences can also be useful outcomes of a project. On the other hand, software development should not be the primary objective. The software is a tool to enhance communication and interaction between different disciplinary teams. It should be a focus of the project primarily only in so far as it encourages communication of ideas and enhances understanding of the integrated nature of the problem.

\section{Conclusions}

Some broad conclusions can be drawn from the situations described above:

\subsection{Targeted modelling}

This research has clearly demonstrated that modelling, as used in IA, must be targeted to the issues, stakeholders and users in order to optimise its chance of success. This is most easily achieved through stakeholder participation throughout the whole project. A tool developed in isolation from stakeholders may not represent their concerns or yield useful outputs, reducing its value. Also, a lack of involvement of stakeholders in the IA process is likely to engender animosity and risk the tool reaching a complexity beyond the users' capabilities and beyond what is needed to achieve the desired outcomes.

\subsection{Politics, conflicts and participatory processes}

The stakeholders involved in the participatory process will inevitably bias the resultant tool. If one group voices its opinion more loudly, or is able to present its opinion better and so is more easily understood, it is likely that the tool will be biased towards its interests. At best this bias will result in an inequitable outcome. If that group has insufficient political and community backing, it may lead to management advice which cannot be adopted or will exacerbate conflict. 


\subsection{Adoption of participatory tools}

\subsubsection{Software}

The software developed for effective decision support tools must present the results so they can be easily viewed and interpreted by the users. The tool must be targeted to the inputs available, outputs necessary and the goals identified by the future users. Contrary to common thinking, the more integration represented in the model, the simpler the model needs to be to allow for testing. Finally the model should not be developed to become a final, fixed product. The tool should be capable of being reused and easily developed in the future to allow for social, economic and physical changes.

\subsubsection{Doing integrated assessment and modelling}

Several integration frameworks already available have demonstrated potential to provide strong support for decision making. For example, at one extreme Bayesian networks (Section 7.3) allow interest groups to conceptualise their system and populate it with a wide variety of knowledge types and sources. At another extreme, coupled component models (Section 7.1) are most useful where users needs to overlay a hydrological network on their system to facilitate decisions at defined spatial locations, and where one has resources to add complexity to the system over time as confidence is gained. It is now timely to accelerate our application of such frameworks and synthesise the scientific and practical lessons from them.

Acknowledgements We are grateful for the funding of the three IA projects discussed in the paper by the Cotton Research and Development Corporation, the NSW Environmental Trust and the NSW Department of (Infrastructure Planning and) Natural Resources. We are also indebted to our colleagues in the various agencies and industry groups and in the wider community for their generous collaboration and input to the projects.

\section{References}

Allan C, Curtis A (2003) Learning to implement adaptive management. Nat Res Man 6:23-28

Borsuk ME, Stow CA, Reckhow KH (2004) A Bayesian network of eutrophication models for synthesis, prediction and uncertainty analysis. Ecol Mod 173:219-239

Bouzaher A, Lakshminarayan PG, Cabe R, Carriquiry A, Gassman PW, Shogren JF (1993) Metamodels and nonpoint pollution policy in agriculture. Water Res 29:1579-1587

Brown DG, Page SE, Riolo R, Rand W (2004) Agent-based and analytical modelling to evaluate the effectiveness of greenbelts. Environ Mod Software 19:1097-1109

Davis JR (1995) Expert systems and environmental modeling. In: Jakeman AJ, Beck MB, McAleer MJ (eds) Modelling change in environmental systems. Wiley, Chichester, pp 505-517

Deaton ML, Winebrake JJ (1999) Dynamic Modelling of Environmental Systems. Springer, New York

Forsyth R (1984) The expert systems phenomenon. In: Forsyth R (ed) Expert systems: principles and case studies. Chapman and Hall, London, pp 3-8

Hare M, Letcher R, Jakeman AJ (2003) Participatory modelling in natural resource management: a comparison of four case studies. Integrated Assessment 4:62-72

Haslam SA, Eggins RA, Reynolds KJ (2003) The ASPIRe model: actualising social and personal identity resources to enhance organisational outcomes. J Occup Organ Psychol 76:83-113

Holling CS (ed) (1978) Adaptive Environmental Management and Assessment. Wiley, Chichester

Jakeman AJ (1989) Application of identification and system parameter estimation to environmental problems: some recent examples. In: Barker HA, Young PC (eds) 7th IFAC symposium on identification and system parameter estimation. York, 3-7 July 1985, 1, Oxford:pergamon Press, pp 445-450

Jakeman AJ, Letcher RA (2003) Integrated assessment and modelling: features, principles and examples for catchment management. Environ, Mod Softw 18:491-501 
Jakeman AJ, Letcher RA, Norton JP (2006) Ten iterative steps in development and evaluation of environmental models. Environ Mod Softw 21:602-614

Jasanoff S (1993) Bridging the two cultures of risk analysis. Risk Anal 13:123-129

Kammen DM, Hassenzahl DM (1999) Should we risk it? Exploring environmental, health and technological problem solving. Princeton University Press, Princeton, N.J., 404 pp

Kidd AL (1987) Knowledge acquisition an introductory framework. In: Kidd AL (ed) Knowledge acquisition for expert systems: a practical handbook. Plenum Press, New York, 194 pp

Letcher RA, Jakeman AJ (2003) Application of an adaptive method for integrated assessment of water allocation issues in the Namoi River Catchment, Australia. Integr Assess 4:73-89

Letcher RA, Croke BFC, Jakeman AJ, Merritt WS (2006) An integrated modelling toolbar for water resources assessment and management in highland catchments: model discription. Agricultural Systems 89:106-131

Letcher RA, Jakeman AJ Croke BFW (2004) Model development for integrated assessment of water allocation options. Water Resour Res 40:W05502, 15 pp

Letcher RA, Jakeman AJ (in press) Integrative frameworks for natural resource management. Envron Mod Softw

Merritt WS, Croke BFW, Jakeman AJ, Letcher RA, Perez P (2004) A biophysical toolbox for assessment and management of land and water resources in rural catchments in Northern Thailand. Ecol Mod 171:279-300

Mostert E (2006), Participation for sustainable water management. In: Giupponi C, Jakeman A, Karssenberg D, Hare M (eds) Sustainable management of water resources: an integrated approach. Elgar, pp. 153-176

Newham LTH, Letcher RA, Jakeman AJ, Kobayashi T (2004) A framework for integrated hydrologic, sediment and nutrient export modelling for catchment-scale management. Environ Mod Softw 19:1029-1038

Newham LTH, Letcher RA, Jakeman AJ (2004b) Integrated assessment and modelling for catchment management: An Australian case study in participation. In: Henze M (ed) Proceedings of international symposium: uncertainty and precaution in environmental management. Copenhagen

Newham LTH, Ticehurst JL, Rissik D, Letcher RA, Jakeman AJ, Nelson P (2004c) Assessing the sustainability of NSW coastal lakes using a Bayesian decision network approach. In: Proceedings NSW coastal conference: seachange? The Delicate Balancing Act. Lake Macquarie 9th-12th November, pp 63-69

Norton JP, Nathan R, Podger G, Vertessy R (2003) Sensitivity assessment needs of complex simulation models for integrated catchment management. In: Post DA (ed) Proceedings modelling and simulation society of Australia and New Zealand. MODSIM 2003, 4:1667-1672

Pearl J (1988) Probablistic reasoning in intelligent systems: networks of plausible inference. Morgan Kaufmann Publishers, Inc. San Francisco, ISBN 1-55860-479-0

Refsgaard JC, Henriksen HJ, Harrar WG, Scholten H, Kassahun A (2005) Quality assurance in model based water management - review of existing practice and outline of new approaches. Environ Mod Softw (in press)

Sadoddin A, Letcher RA, Jakeman AJ (2003) A Bayesian decision network approach for salinity management in the Little River Catchment. NSW, In: Post DA (ed) Proceedings modelling and simulation society of Australia and New Zealand. MODSIM 2003, 3:953-958

Schmoldt DL (1998) Knowledge acquisition using linguistic-based knowledge analysis. AI Appl 12(1-3):1-20

Sell P (1985) Expert systems: a practical introduction. John Wiley and Sons, Inc, New York

Spate JM, Croke BFC, Jakeman AJ (2003) Data mining in hydrology. In: Post DA (ed) Proceedings modelling and simulation society of Australia and New Zealand. MODSIM 2003 4:422-427 